\title{
La coyuntura internacional en 2008 y 2009
}

\author{
Dra. Beatriz Herrera García
}

Docente Principal de la Facultad de Ciencias Contables

\section{RESUMEN}

Uruguay (11.5\%), Perú (9,4\%) y Panamá (9.2\%) lideraron el crecimiento económico latinoamericano en el 2008. La región latinoamericana en su conjunto creció 4.6 por ciento, completando su sexto año consecutivo de expansión. Sin embargo, por efecto de la grave crisis mundial se pronostica una brusca desaceleración del crecimiento en 2009, se estima tan solo una tasa de 1.9 por ciento, que provocará una caída del ingreso regional por habitante aumentando los niveles de pobreza.

A diferencia de las crisis mundiales anteriores en las que la deuda pública externa era el principal factor desestabilizador, en la crisis actual es el sector privado el más expuesto a la volatilidad cambiaria. Por lo que las empresas privadas endeudadas en moneda extranjera están sufriendo un impacto negativo en sus balances. Los casos más emblemáticos son Chile (9.0\% del PBI), Perú (7.9\% del PBI) y Argentina (3.8\% del PBI).

Palabras clave: Crisis financiera mundial, desaceleración económica, crisis en los mercados financieros. 


\section{INTRODUCCIÓN}

La crisis financiera mundial es la peor crisis desde 1930, caracterizada por una profunda fisura del sistema financiero mundial, la caída vertiginosa de los mercados de valores y un congelamiento crediticio generalizado. El agravamiento de estos factores ha dado lugar a una crisis económica mundial de gran envergadura en 2008 y 2009. En ese escenario, las perspectivas para las economías en vías de desarrollo se tornan sombrías, incluso para aquellas economías como la peruana que han tenido un sólido desempeño.

Según los pronósticos basados en el Proyecto LINK, se estima que el producto y el comercio mundial decrecerán bruscamente en 2009 (ver cuadro 1.1), lo cual significará una brusca caída del ingreso mundial por habitante. El resultado será un decrecimiento del producto mundial como consecuencia de la caída del crecimiento en las economías desarrolladas $(-1.5 \%)$, de una singular desaceleración del crecimiento en las economías en transición $(2.7 \%)$ y en las economías en vías de desarrollo (2.7\%). Estos resultados están condicionados por la dimensión y prolongación de la contracción del crédito global y de la improbable restauración del sistema financiero en 2009. En consecuencia, el tamaño de la economía mundial se reduciría y la pobreza mundial se agravaría. Es decir, el impacto de la crisis mundial derivada de la pérdida de riqueza financiera y no financiera del sector privado se traducirá en una drástica reducción del crédito que afectará a los niveles de inversión y consumo como efecto de la restricción de la liquidez. Las economías latinoamericanas histórica y altamente dependientes de los factores exógenos verán afectados sensiblemente sus tendencias económicas actuales; se agudizarán sus asimetrías, mientras las tasas del PBI diminuyan aceleradamente por la caída de las exportaciones, las inversiones y el consumo, las tasas de desempleo aumentarán crecientemente debido a que en el mercado laboral la demanda de trabajo será altamente sensible a los efectos de la crisis.

La profundidad y duración de la crisis mundial dependerá de los cambios y de las políticas económicas anticrisis que implementen las autoridades de las economías desarrolladas para detener la caída del gasto privado y la restricción crediticia y, por otro lado, sus bancos centrales de reserva consigan contener el riesgo sistémico.

\section{BREVE HISTORIA DE LA CRISIS}

El año 2008, marca el final de un período con escasos precedentes en la historia económica de América Latina. El brusco cambio de ten-

Cuadro 1.1

CRECIMIENTO MUNDIAL DEL PBI, 2006-2009 (en porcentaje)

\begin{tabular}{|l|c|c|c|c|}
\hline & 2006 & 2007 & 2008 & $2009^{*}$ \\
\hline Producto Mundial & 4.0 & 3.8 & 2.5 & -0.4 \\
-Economías desarrolladas & 2.9 & 2.5 & 1.1 & -1.5 \\
-Economías en transición & 7.8 & 8.3 & 6.9 & 2.7 \\
-Economías en desarrollo & 7.0 & 9.1 & 5.9 & 2.7 \\
-Economías latinoamericanas & 5.8 & 5.7 & 4.6 & 1.9 \\
\hline Comercio Mundial & 8.8 & 6.3 & 4.4 & -3.1 \\
\hline
\end{tabular}

* Proyecciones: Proyecto LINK. / FuENTES: UN/DESA, CEPAL, 2008. 
dencia es resultado de los efectos de la crisis mundial que se agudizó a mediados del año 2007. Inicialmente se presentó como un problema del mercado de hipotecas de alto riesgo (subprime) en los Estados Unidos, proceso que ha ido agudizándose hasta convertirse en una crisis sistémica, afectando a los mercados de crédito de las economías desarrolladas.

Así, el enfriamiento de la actividad económica mundial (caída de las exportaciones mundiales del 17.1 por ciento en 2007 al 4.7 por ciento en 2008) se originó en los problemas derivados de la crisis financiera que estalló a mediados de 2007 y fue agravándose en adelante a pesar de los esfuerzos de las autoridades monetarias del FED norteamericano. En el epicentro de la crisis (conjunto de países desarrollados), la economía creció tan solo 1,1 por ciento en 2008 , frente al 2,5 por ciento del 2007, siendo ambas tasas bajas en relación a sus capacidades; lo peor de la crisis aún no ha llegado por lo que se pronostica una fuerte contracción en 2009 (-1.5\%).

En efecto, el primer factor deflacionario de la crisis financiera internacional se presentó como una rápida desvalorización de los activos inmobiliarios en los Estados Unidos. El nivel de los precios empezó a disminuir a principios de 2007, y a mediados de 2008 el nivel del índice representativo del precio de las viviendas se situaba un 17.7 por ciento debajo del mismo período del año anterior. Este proceso deflacionario ejerció presiones sobre el balance de los deudores hipotecarios y de diversas entidades financieras. Los esquemas de refinanciamiento de los intermediarios se vieron obstaculizados por problemas crecientes de morosidad y por la desvalorización de los activos de respaldo, con la consecuente elevación abrupta de la carga relativa de pasivos. A mediados de 2007, se evidenció que se había producido una severa descapitalización de diversos activos de inversión pertenecientes a importantes instituciones financieras internacionales, cerrándose las posibilidades de endeudamiento de dichas instituciones en mercados interbancarios y de corto plazo.

El segundo factor deflacionario se presentó como la depreciación de los activos bursátiles. El nivel del índice Dow Jones (representa a las 30 acciones industriales más representativas de EE.UU.), se situó a mediados de noviembre de 2008 en un nivel menor que el de julio de 2007 ( los valores cayeron un 35,4\%).

Un tercer factor deflacionario surge a mediados de $2008^{1}$, a partir de las tendencias recesivas de Japón y de países de la zona del euro, y de la apreciación del dólar: la caída de los precios de los productos básicos o primarios (commodities).

En suma, en la primera etapa del desencadenamiento de la crisis, los flujos privados de financiamiento del déficit de la cuenta corriente de la balanza de pagos de los Estados Unidos se redujeron notablemente. Entre 2003 y 2007 representaron, en promedio, un 2.6 por ciento del PBI norteamericano, mientras que en el lapso entre el cuarto trimestre de 2007 y el segundo trimestre de 2008 alcanzaron apenas un 0.6 por ciento del PBI, dejando el financiamiento de la brecha externa en función de los flujos oficiales norteamericanos, que cubrieron aproximadamente el 90 por ciento del saldo total.

Aunque los desequilibrios macroeconómicos globales se han reducido en alguna medida a causa de la recesión que atraviesan las economías desarrolladas, el déficit

1 Un acontecimiento importante en el desarrollo de la crisis financiera internacional fue la quiebra de Lehman Brothers. 
externo de los Estados Unidos se encuentra aún muy alto y su balance financiero externo negativo no deja de crecer. La tendencia hacia la depreciación del dólar que se viene experimentando desde 2002 parece reflejar la percepción generalizada de que un déficit tan alto de la balanza comercial de los Estados Unidos, y su consecuente acumulación neta de pasivos internacionales, es insostenible. La tendencia reciente marcada por la búsqueda de la seguridad en el dólar no ha hecho sino aumentar aún más el grado de endeudamiento de los Estados Unidos. De allí que el peso del endeudamiento pueda precipitar un nuevo derrumbe del dólar una vez que el proceso de desapalancamiento de agentes financieros toque a su fin.

La teoría económica sostiene que en el fondo de la crisis mundial está el patrón de crecimiento global configurado a partir de la caída del Muro de Berlín. Tal proceso insostenible se ha basado en una fuerte demanda de bienes de consumo en los estados Unidos, estimulada con crédito fácil otorgado al grupo de alzas explosivas en los precios de las viviendas. Dicha demanda ha tenido como contrapartida el crecimiento exportador y de demanda de inversión en algunas economías en desarrollo, siendo China el caso más notable. Los déficits crecientes de los Estados Unidos se han financiado con superávits comerciales de economías como China, Japón y Rusia, que han acumulado grandes cantidades de reservas internacionales, contribuyendo a la demanda mundial de activos denominados en dólares.

\section{EFECTO DE LA CRISIS EN LOS MERCADOS FINANCIEROS}

Con la intensificación de la crisis en setiembre de 2008, las autoridades mostraron in- terés en un nivel de coordinación internacional para enfrentar la crisis. Dicho cambio de actitud ha alterado el panorama de desregulación financiera que prevalecía hasta entonces. Se dispusieron gigantescos fondos públicos para recapitalizar bancos, así como provisión de avales gubernamentales sobre los depósitos bancarios. Otros gobiernos también han empezado a proponer paquetes fiscales y monetarios de reactivación para evitar una catástrofe mundial.

Dichas medidas se han orientado a restaurar la confianza y desbloquear los mercados de crédito y de dinero utilizando fondos y garantías públicas. Sin embargo, a finales de 2008 importantes segmentos del mercado crediticio se mantienen bloqueados.

A finales de 2008 , se observó una desaceleración seguida de una caída de los flujos de cartera, incrementándose los costos del financiamiento internacional. Por lo que la actividad financiera de la región enfrentó los problemas sufridos en los mercados interbancarios y el impacto en los mercados de créditos locales de la restricción de crédito externo, que constituyen las dos vías a través de las cuales los efectos de las turbulencias de los mercados financieros de las economías desarrolladas pueden trasladarse a la región.

Ante este panorama, es de especial preocupación la evolución de las condiciones de acceso al crédito internacional de un conjunto de grandes empresas latinoamericanas, que habitualmente se financian en los mercados financieros internacionales. Ante la situación actual y las perspectivas futuras, es espera una mayor dificultad para cubrir las necesidades de financiamiento. La menor disponibilidad de financiamiento externo llevará a las empresas privadas de la región a recurrir al mercado interno para financiar sus actividades propias y sus obligaciones externas (pago de sus intereses y amortizaciones), 


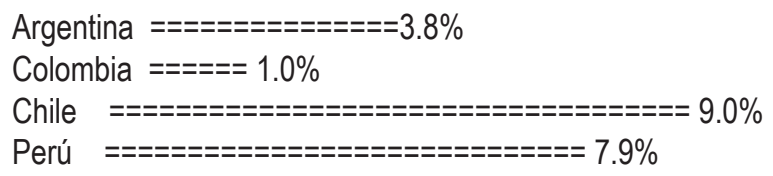

FuentE: CEPAL, 2008.

lo que provocará mayores dificultades de acceso a recursos financieros para las pequeñas y medianas empresas.

A diferencia de crisis anteriores en las que la deuda externa pública era el principal factor desestabilizador, en la crisis actual, en muchos países (ver, gráfico 1.1), esta vez es el sector privado el más expuesto a la volatilizada cambiaria. Por lo que las empresas privadas endeudadas en moneda extranjera están sufriendo el impacto negativo sobre sus balances, derivado de las devaluaciones aceleradas de varias monedas en la región.

Tal como se afirmó, la crisis internacional ha provocado un endurecimiento de las condiciones financieras internacionales afectando a los flujos de inversión extranjera directa (IED) que han sido una fuente importante de recursos en los últimos años, asimismo, representan una restricción importante para que inversionistas privados de la región accedan a créditos externos como en el pasado reciente.

Los sistemas financieros de los países en desarrollo han visto aumentar sus riesgos a través de otros canales, debido a que los inversionistas extranjeros en países en desa- rrollo han empezado a retirar sus fondos de dichas economías en sus esfuerzos por reducir el grado de apalancamiento financiero. Con los ajustes del crédito en los mercados globales, los costos de financiamiento en los mercados de las economías en desarrollo han subido, tal como se puede apreciar en los márgenes de tasa que mide el $\mathrm{EMBI}^{2}$, la subida reciente de dicho índice en los márgenes ha sido uniforme, lo que sugiere un alto grado de contagio en la aversión de los inversionistas con respecto a las economías en desarrollo en su conjunto. Se prevé que los márgenes se mantendrán altos en el 2009.

\section{EFECTO DE LA CRISIS EN LAS REMESAS DE LOS MIGRANTES INTERNACIONALES}

Como se advirtió (supra), en el 2009 se espera una caída de la tasa de ocupación y un aumento del desempleo en las economías desarrolladas. Es lógico suponer que la pérdida del empleo afecta a los hogares de bajos ingresos, por la pérdida de una proporción del ingreso del hogar, asimismo, los miembros

2 Emerging Markets Bond Index. Dicho índice calcula la diferencia entre los tipos de interés de los centros emisores y los que efectivamente cobran las instituciones financieras prestamistas en las economías emergentes. 
de los hogares más pobres suelen insertarse en categorías de ocupación y ramas de actividad que se diferencian de las de los ocupados provenientes de hogares más pudientes. Por ejemplo, en las ciudades, los ocupados que forman parte del primer y segundo quintil representan el 17.1 por ciento y el 22.2 por ciento, respectivamente, de los trabajadores del sector construcción ${ }^{3}$. Dado que en muchos países el enfriamiento de la economía tiene notorias repercusiones en este sector, se puede suponer que muchos hogares que ya perciben ingresos bajos sufrirán una importante pérdida de bienestar como efecto de la crisis.

Por otro lado, en términos de categoría de ocupación, los quintiles de bajos ingresos suelen estar sobre representados en el empleo doméstico, en el trabajo por cuenta propia y en el trabajo no remunerado. La ocupación en la categoría empleo doméstico estaría amenazado por situaciones de crisis, ya que muchos hogares de los estratos medios que emplean personal para servicio doméstico tienden a despedirlas ante la pérdida de ingresos o la amenaza de que algún miembro del hogar pierda su empleo. En las otras categorías: trabajo por cuenta propia y trabajo no remunerado, el número de trabajadores suelen aumentar en momentos de bajo crecimiento económico debido a que este tipo de trabajo se percibe como la única manera de generar ingreso o de contribuir a él (es el caso de la última categoría).

En consecuencia, si bien en muchos estudios se ha puesto énfasis que los emigrantes internacionales, en general, no provienen de los hogares más pobres, las remesas que envían desde el exterior suelen tener un impacto positivo en la distribución, pues favorecen a hogares de ingreso bajo y mediobajo. Por lo que la caída de las remesas como consecuencia de la crisis de las economías desarrolladas, destino de las migraciones, junto con la implementación de políticas de control migratorio más severas, tiene un claro impacto negativo en el bienestar de estos hogares.

En otras palabras, dada la profundidad de la crisis económica mundial, el menor dinamismo del empleo y el aumento del desempleo en las economías desarrolladas incidirá en la cantidad de las remesas que envían los trabajadores emigrantes a sus familias en sus países de origen. Esta ha sido una fuente muy apreciable de ingresos externos que ha contribuido a generar el bienestar de familias de bajos ingresos, por

GRÁFICO 1.2

TRANSFERENCIAS CORRIENTES (REMESAS RECIBIDAS), 2007

(en millones de dólares)

010002000300040005000600070008000 millones de dólares

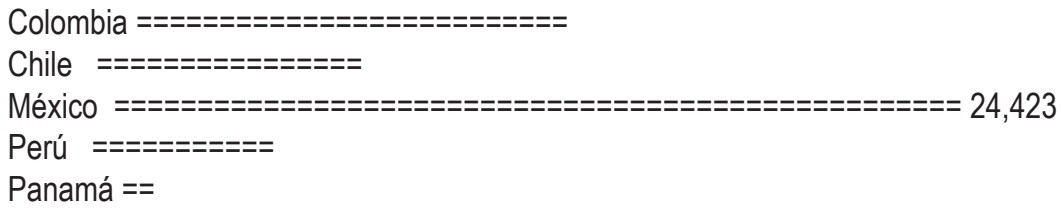

FUENTE: CEPAL, 2008.

3 Además, los empleos de los miembros de los hogares más pobres suelen concentrarse en el sector informal, de manera que en caso de pérdida del empleo no cuentan con mecanismos de protección. 
lo que su disminución afectará con creces a este grupo socioeconómico.

Son varios los países de la región afectados por la reducción drástica de las remesas del exterior, que también de alguna manera se verá reflejado en sus balanzas de pagos internacionales (infra).

\section{EFECTO DE LA CRISIS EN LOS PRECIOS DE LOS COMMODITIES Y EN LOS TERMINOS DE INTERCAMBIO}

La grave crisis económica de las economías desarrolladas y la significativa desaceleración del crecimiento en las economías emergentes (China, India, etc.), tienen un efecto negativo en la demanda y los precios de las exportaciones latinoamericanas. La importancia de ese efecto es debido a que en gran parte de los países de la región, los productos básicos o primarios (commodities) representan una porción significativa de la canasta de exportaciones y constituyen una significativa fuente de recursos públicos.

El efecto se apreciará como una caída del precio de los productos básicos o commodities derivada de la brusca desaceleración del crecimiento mundial e implicará un deterioro de los términos de intercambio para la región en su conjunto.

Es de notar que el ritmo de crecimiento de estos precios ha aumentado de forma regular desde mediados de 2007 hasta mediados de 2008. Esta evolución favorable es aplicable a todos los productos básicos, pero es particularmente notable en el petróleo y en algunos metales como el cobre, oro y en bienes alimenticios como la soja, maíz y trigo.

Tomando como base en año 2000 (2000 $=100$ ), el índice de precios de los productos básicos alcanzó un valor máximo entre junio y agosto de 2008. Así, los precios se dispararon con un "pico" en julio: los minerales y metales alcanzaron un máximo de 390 por ciento; los alimentos alcanzaron un máximo de 280 por ciento, y el petróleo alcanzó un máximo de 470 por ciento. Luego, a partir de ese momento se registraron fuertes caídas, que reflejan la fase recesiva y deflacionaria de la crisis económica mundial. A fines de 2008, los índices de precios habían retrocedido fuertemente (minerales y metales alcanzaron $160 \%$ —precios similares a los de fines de 2005-; alimentos alcanzaron $155 \%$ y el petróleo alcanzó 130\% — precios equivalentes a los precios de fines de 2004-).

Para todos los países de la región que habían aprovechado de la mejora de sus términos de intercambio, observada desde 2003, la evolución pronosticada de los precios de los productos básicos (2009) implicará la detención de uno de los motores del crecimiento en los últimos cinco ańos. En efecto, se estima una caída del 7.8 por ciento.

Para Chile y Perú, exportadores de metales se esperan caídas en los términos de intercambio en torno al 8,0 por ciento en 2008, en torno al 30 por ciento en 2009, que representan las caídas más significativas de toda la región.

\section{EFECTO DE LA CRISIS EN EL COMERCIO EXTERIOR}

Durante el primer semestre de 2008, las exportaciones de la región tendieron a incrementarse con ritmos crecientes como resultado de la coyuntura alcista por la que atravesaban varios mercados de productos básicos (petróleo, minerales, alimentos y materias primas agropecuarias). A su vez, los aumentos de precios, aunados al dinamismo de la actividad interna y las apreciaciones 
cambiarias, dieron más vigor a una aceleración de las importaciones que se había iniciado en el 2007.

En la segunda mitad de 2008, las exportaciones se vieron afectadas por el desplome de los precios de los productos básicos; al mismo tiempo, las importaciones se desaceleraron en la medida que cedieron las presiones inflacionarias externas y se debilitaron los ritmos de actividad interna. Sin embargo, en el 2008 se produjo una aceleración del ritmo de crecimiento del valor de las exportaciones (18.3\%) y del valor de las importaciones $(23.0 \%)^{4}$.

El efecto de estos movimientos en la balanza comercial regional fue un saldo favorable (saldo positivo en el 2008), con tendencia a la baja del superávit comercial en relación al año anterior (ver, cuadro 1.2).

Sin embargo, de forma sorprendente la cuenta corriente de la región en su conjunto ${ }^{5}$, por primera vez en cinco años, registró un déficit significativo (de -27177 millones de dólares).

El deterioro de la cuenta corriente muestra un deterioro en toda su estructura (balan- zas: comercial, rentas y transferencias), observándose una asimetría en el comportamiento de la balanza de bienes cuya reducción del saldo positivo contrasta con el aumento del saldo negativo de la balanza de rentas. En el primer caso, el resultado surge de la aceleración en las importaciones, mientras, que en el segundo se relaciona con el con un salto en el aumento del pago netos de rentas.

En el 2008, los pagos por intereses y utilidades del capital extranjero habían ascendido a 21.5 por ciento más que en el año 2007 , lo que explica principalmente el resultado alcanzado en la cuenta corriente de la región. En los casos de Perú, Chile y Colombia, se aprecia la misma tendencia.

En el saldo positivo de las transferencias corrientes de gran relevancia para algunos países (ver gráfico 1.2), se observa una desaceleración en relación a la tendencia de años anteriores. Esto refleja un progresivo debilitamiento del envío de remesas por parte de los trabajadores emigrados vinculado a las condiciones recesivas de las economías desarrolladas.

CUADRO 1.2

LA CUENTA CORRIENTE DE LA BALANZA DE PAGO 2007 Y 2008

\begin{tabular}{|l|l|l|l|l|}
\hline \multicolumn{1}{|c|}{} & $\begin{array}{l}\text { (en millones de dólares) } \\
\text { Balanza Comercial } \\
2007 \quad 2008\end{array}$ & $\begin{array}{l}\text { Balanza de Rentas } \\
20072008\end{array}$ & $\begin{array}{c}\text { Transferencias } \\
20072008\end{array}$ & $\begin{array}{c}\text { Cuenta Corriente } \\
20072008\end{array}$ \\
\hline América Latina & 4769419353 & $-92954-112666$ & 6454566136 & $19285-27177$ \\
Perú & $7,4291,656$ & $-8,418-9,600$ & $2,4952,869$ & $1,505-5,076$ \\
Chile & 224918,361 & $-18265-17000$ & $2,9743,000$ & $7,200-5,639$ \\
Colombia & $-3,203-1,081$ & $-7,886-10784$ & $5,2315,422$ & $-5,859-6,442$ \\
\hline
\end{tabular}

Fuente: CEPAL, 2008.

4 Por lo que en el 2008, los términos de intercambio continuaron mejorando en un 4.6 por ciento, debido a la evolución relativa de los precios de las exportaciones y de las importaciones (llegando a representar un estimado de 43,600 millones de dólares).

5 La cuenta corriente o balanza de pagos en cuenta corriente es la parte más importante de una balanza de pagos, y es resultado de la sumatoria de la balanza comercial, , balanza de rentas (pagos al exterior por concepto de los intereses y utilidades del capital extranjero) y las transferencias. 


\section{CONCLUSIONES}

Según Bárcena (2008), por efecto de la crisis económica internacional uno a uno se estarían apagando los factores (motores) del crecimiento económico latinoamericano. Los motores del crecimiento durante los últimos cinco ańos, tanto en el sector real como en el financiero se identifican como:

- La aceleración de las exportaciones.

- El aumento del precio de los commodities.

- El aumento de las remesas provenientes del exterior.

- Los mejores ingresos por turismo.

- El incremento de los flujos de inversión extranjera directa.

- La disminución del costo del crédito internacional.

- El aumento de la disponibilidad de financiamiento externo.

Según Rob Vos (2008), aunque sean los países desarrollados los que originaron la grave coyuntura recesiva mundial, la debilidad económica ha empezado a afectar drásticamente a los países en desarrollo como a los países en transición, dando lugar a perspectivas de decrecimiento global acelerado en 2009.

Entre los países en desarrollo, África, será afectado por el impacto de la desaceleración global a través de la debilidad de la demanda de las exportaciones, bajos precios de los productos básicos y la reducción de los flujos de inversión. Asia del Este, decaerá por una reducción significativa de sus exportaciones y experimentará pérdidas financieras debido a su alta exposición a los mercados financieros globales. Asia del Sur, la caída de su actividad económica se manifestará en tanto la desaceleración industrial se transmita hacia el sector terciario. Asia
Occidental, será afectada al precipitarse los ingresos por exportaciones petroleras y caer el gasto de inversión.

El crecimiento en América Latina sufrirá una caída considerable debido a la disminución de los precios de los productos básicos de exportación y las restricciones crediticias internacionales.

En suma, la crisis financiera internacional, que se suma a la crisis energética y a la crisis alimentaría, provocará un grave retroceso en relación a la reducción de la pobreza. Asimismo, las restricciones crediticias y el debilitamiento del crecimiento reducirán los ingresos públicos, afectando la capacidad de inversión pública en los países en desarrollo. Si no se establecen redes de protección social efectiva, serán "los pobres de la tierra" los más golpeados por la crisis económica mundial. Se estima que 125 millones de personas de los países en desarrollo han caído en condiciones de extrema pobreza a consecuencia del alza internacional de los precios de los alimentos desde el 2006.

\section{REFERENCIAS}

Bárcena, A. Presentación del balance Preliminar de las Economías de América Latina. Santiago de Chile, diciembre 2008.

CEPAL. Balance Preliminar de las Economías de América Latina y el Caribe 2008, Chile.

FMI. World Economic Outlook. 2008, Washington D.C.

FMI. Perspectivas de la Economía Mundial. 2009, Washington D.C. 
UN/DESA. Cómo superar la inseguridad económica, estudio económico y social, 2008, Nueva York.

UN/DESA. "Overcoming Economic Insecurity", World Economic and Social Survey, 2008, New York.

UN/DESA. World Economic Situation and Prospects 2009, Global Outlook 2009, New York.
UN/DESA. Situación y perspectivas para la economía mundial, 2009, New York.

Rов Vos. Advance Release, World Economic Situation and Prospects 2009, Doha, Qatar, December.

Revista América Economía, varios números.

Revista Newsweek, varios números.

Diario La República, varios números.

Diario Gestión, varios números. 